\title{
Kinetic investigation of oxidation of aromatic anils by magnesium monoperoxyphthalate in aqueous acidic medium
}

\author{
Venkatesh Ramakrishnan, Karunakaran Kulandaivelu* \\ Sona College of Technology (Autonomous), Department of Chemistry, Salem - 636005, Tamilnadu, India \\ *Corresponding author: e-mail: karunachemist@gmail.com
}

\begin{abstract}
The kinetics of oxidation of aromatic anils to benzaldehyde and azobenzene by magnesium monoperoxyphthalate (MMPP) has been studied in aqueous acetic acid medium. The low dielectric constant of the medium facilitates the reactivity. It has been found that ionic strength of the reaction has no significant effect on rate. The added acrylonitrile has no effect on the reaction rate indicating the absence of free radical mechanism. The added $\mathrm{Mn}$ (II) decreases the rate of the reaction, which indicates the involvement of two-electron transfer. Highly negative $\Delta \mathrm{S}^{\#}$ values indicate a structured transition state. The deviation of Hammett plot is noted and a non-linear concave downward curve is obtained for the anils with substituents in the aniline moiety. The observed break in the $\log \mathrm{k}_{\mathrm{obs}}$ versus $\sigma$ is attributed to the transition state whereas the non-linear concave upward curve is observed for the substituents in the benzaldehyde moiety and a non-linear concacve upward curve is observed for the substituents in the combination of aniline and benzaldehyde moiety and a suitable mechanism is proposed.
\end{abstract}

Keywords: : Aromatic anils, Kinetics, Oxidation, Magnesium monoperoxyphthalate.

\section{INTRODUCTION}

MMPP is a commercially available, inexpensive and relatively stable oxidant. Since its development in the early $80 \mathrm{~s}$, it has been used in the oxidation of various functional groups ${ }^{1,2}$, for instance, in the epoxidation of alkenes, sulfur and nitrogen oxidations, Baeyer-Villiger oxidation $^{\mathbf{1}, 3}$ as well as in the oxidative cleavage of hydrazones $^{3}$. One of the reasons that have limited large applications of MMPP is its low solubility in non-polar solvents. Reactions using MMPP have usually been carried out in water or low molecular weight alcohols as solvents. For insoluble substrates in polar solvents, reactions have been conducted in biphasic media combined with a phase transfer catalyst. However, reactions in ethanol or phase transfer media are often not efficacious. An attempt to overcome these limitations has been the use of a solid-phase-supported version of MMPP on wet silica gel or moist alumina ${ }^{4}$. Among the oxygen donars, towards oxidation, MMPP was recently found to be a very efficient oxidant ${ }^{5-8}$. In this paper, we report simple and effective approaches for the selective aromatic anils using MMPP in organic solvents. Aromatic anils, $\mathrm{X}-\mathrm{CH}=\mathrm{N}-\mathrm{Y}$ have two phenyl rings $\mathrm{X}$ and $\mathrm{Y}$, the ring $\mathrm{X}$-originates from the aromatic benzaldehyde moiety and $\mathrm{Y}$ from aniline moiety. It is possible to evaluate the reaction constant (@) from the Hammett plot by varying the substituents in one of the rings X or Y, and keeping the same substitutent in the other ring. It is therefore possible to get several reaction constants for different substituents in one of the two rings. The kinetics and mechanism of oxidation of some substituted anils, using several oxidants have been reported ${ }^{9-12}$. Literature survey reveals that no report is available on the kinetics of oxidation of meta- and para- substituted aromatic anils with MMPP; hence this study has considered it worthwhile to do research on the kinetics and mechanism of oxidation of anils by MMPP.

\section{EXPERIMENTAL}

Anils are prepared ${ }^{\mathbf{1 2}}$ by refluxing equimolar quantities of benzaldehyde and aniline (Sigma Aldrich) in ethanol for about 2 to $3 \mathrm{~h}$. The resulting solution is cooled and poured into cold water. The precipitated anil is filtered off, washed with ethanol and dried. It is recrystallised from ethanol. The purity of the anils is checked by determining their melting points and FT-IR spectrum. All other chemicals used are of analytical grade (Merck, India). Acetic acid is purified by redistillation. All the reagents are prepared just before the reactions are carried out. Aqueous solution of magnesium monoperoxyphthalate (Sigma Aldrich) is prepared fresh and standardized iodometrically ${ }^{13}$. All the reactions are carried out in a thermostat and the temperature is controlled to $\pm 0.1^{\circ} \mathrm{C}$. The reactions are performed under pseudo-first order conditions by maintaining the excess of anil over oxidant. The mixture is homogeneous throughout the course of the reaction. The progress of the reactions is followed by estimating the unreacted oxidant iodometrically at regular time intervals. The rate constants $\left(\mathrm{k}_{\mathrm{obs}}\right)$ are from $\log$ (titre) versus time plots. All the rate constants are averages of two or more determinations. Under kinetic conditions, stoichiometric amounts of the substrate and magnesium monoperoxyphthalate are mixed. After $24 \mathrm{~h}$, the acetic acid is neutralized and the reaction mixture ethanol is extracted. The dark brown extract, when subjected to preparative TLC on silica gel, gives two distinct spots. On evaporation of ethanol the products are found to be benzaldehyde which is identified by the isolation of its 2,4-dinitrophenylhydrazone derivative (m.p. $238^{\circ} \mathrm{C}$, lit. $239^{\circ} \mathrm{C}$ ) and azobenzene identified by its m.p. $67^{\circ} \mathrm{C}$ (lit. $68^{\circ} \mathrm{C}$ ) and UV-VIS spectrum. Formation of the azobenzene is also confirmed by UV-visible spectra $\left[\lambda_{\max } 429 \mathrm{~nm}\right]$ of the reaction solution during and after the completion of the reaction. This was in agreement with the literature value ${ }^{14}$. The $\lambda_{\max }$ of azobenzene is confirmed by comparing with that of the authentic sample in the aqueous acetic acid medium. 
The oxidation reactions of meta- and para-substituents of aromatic anils are studied at different temperatures $(298,303,308,313$ and $318 \mathrm{~K})$ to evaluate various thermodynamic parameters. From the Eyrings equation ${ }^{15}$, the thermodynamic parameters, enthalpy of activation and entropy of activation are figured out from the expression $\mathrm{k}_{\mathrm{obs}}=\left(\mathrm{k}_{\mathrm{b}} \mathrm{T} / \mathrm{h}\right) \exp ^{\Delta \mathrm{G} \# / \mathrm{RT}}$

Where $\mathrm{k}_{\mathrm{b}}$ is Boltzmann's constant, $\mathrm{T}$ is the temperature in Kelvin, $\mathrm{h}$ is plank's constant, $\mathrm{R}$ is the gas constant, $\Delta \mathrm{G}^{\#}$ is the activation of Gibbs free energy, $\Delta \mathrm{H}^{\#}$ is the enthalpy of activation and $\Delta S^{\#}$ is the entropy of activation. $\Delta \mathrm{G}^{\#}$ and energy of activation $\left(\mathrm{E}_{\mathrm{a}}\right)$ can be calculated from the following expressions respectively. $\Delta \mathrm{G}^{\#}=\Delta \mathrm{H}^{\#}-\mathrm{T} \Delta \mathrm{S}$ and $\mathrm{E}_{\mathrm{a}}=\Delta \mathrm{H}^{\#}+\mathrm{RT}$

\section{RESULTS AND DISCUSSION}

The reaction pathway is the second order with respect to the concentration of anil and is the first order with respect to the concentration of MMPP. Under the identical experimental conditions [Anil] $>>[\mathrm{MMPP}]$, the pseudo-first order linear plot of log (titre) versus time is linear upto $90 \%$, depicted in Fig. 1. The plot of $\log \mathrm{k}_{\mathrm{obs}}$ against log [Anil] (Fig. 2), reveals that the order of the reaction with respect to [anil] is two, with a correlation coefficient $r=0.979$. The kinetic order in acidity appears

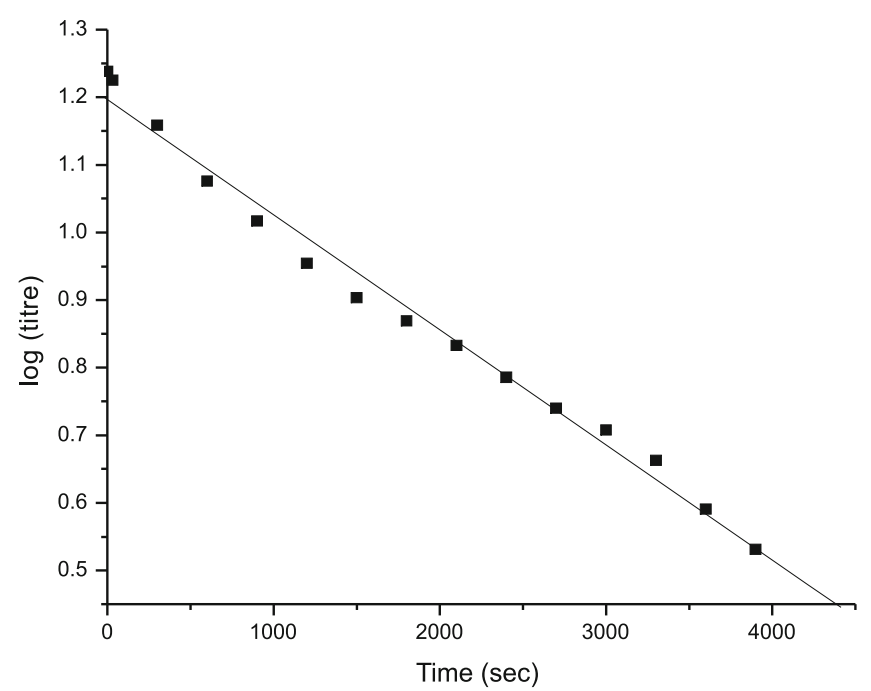

Figure 1. Pseudo-first order plot for MMPP oxidation of aromatic anil at $318 \mathrm{~K}$

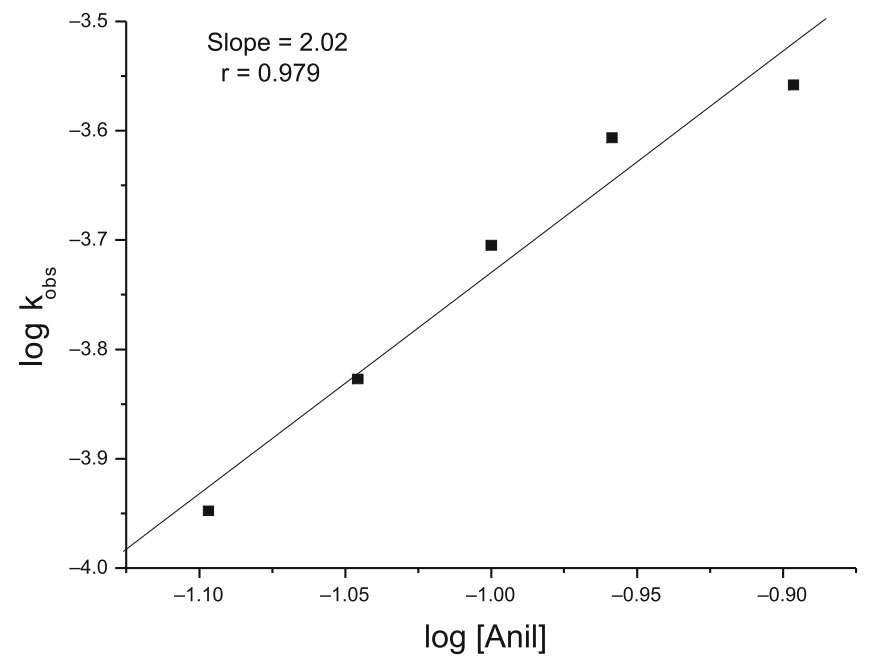

Figure 2. Plot of $\log \mathrm{k}_{\mathrm{obs}}$ Vs $\log$ [Anil] at $308 \mathrm{~K}$ to be united, but increase in $\left[\mathrm{H}^{+}\right]$, decreases the rate of the reaction. The kinetic results are summarized in Table 1. The low dielectric constant of the medium facilitates the reactivity. The ionic strength of the reaction varies by the addition of $\mathrm{Na}_{2} \mathrm{SO}_{4}$ and its influence on reaction rate is studied and it is found that it has no significant effect on the reactivity. No polymerization with acrylonitrile is observed. Furthermore, the rate of conversion is invariant when acrylonitrile is added. Addition of $\mathrm{Mn}$ (II) decreases the rate of the reaction and the rate data are listed in Table 2. Temperature dependence studies are conducted for all the substituted anils (substitution in $\mathrm{X}$ and $\mathrm{Y}$ ring separately) between 298 and $318 \mathrm{~K}$. The rate constants for the reaction systems and activation parameters are evaluated from the Eyring plots as listed in Table 3.

When an attempt is made to fit in the rate data of meta- and para-substituted anils (substitution in aniline moiety and substitution in benzaldehyde moiety separately and substitution in both aniline moiety and benzaldehyde moiety) into the Hammett equation, a non-linear concave downward curve is obtained for the anils with

Table 1. Effect of varying the concentration of Anil, MMPP, $\mathrm{H}_{2} \mathrm{SO}_{4}$ and solvent composition on the rate at $308 \mathrm{~K}$

\begin{tabular}{|l|c|c|c|c|}
\hline $\left.\left.\begin{array}{l}10^{2}[\text { Anil }] \\
(\mathrm{mol} \mathrm{dm}\end{array}\right)^{-3}\right)$ & $\begin{array}{c}10^{3}[\mathrm{MMPP}] \\
\left.(\mathrm{mol} \mathrm{dm})^{-3}\right)\end{array}$ & $\begin{array}{c}{\left[\mathrm{H}^{+}\right]} \\
\left(\mathrm{mol} \mathrm{dm}^{-3}\right)\end{array}$ & $\begin{array}{c}\mathrm{HOAc} \\
(\%)\end{array}$ & $\begin{array}{c}10^{4} \mathrm{k}_{\text {obs }} \\
\left(\mathrm{s}^{-1}\right)\end{array}$ \\
\hline 8.0 & 5.0 & 1.0 & 50 & 1.12 \\
\hline 9.0 & 5.0 & 1.0 & 50 & 1.48 \\
\hline 10.0 & 5.0 & 1.0 & 50 & 1.97 \\
\hline 11.0 & 5.0 & 1.0 & 50 & 2.47 \\
\hline 12.0 & 5.0 & 1.0 & 50 & 2.76 \\
\hline 10.0 & 3.0 & 1.0 & 50 & 1.17 \\
\hline 10.0 & 4.0 & 1.0 & 50 & 1.36 \\
\hline 10.0 & 5.0 & 1.0 & 50 & 1.97 \\
\hline 10.0 & 6.0 & 1.0 & 50 & 1.76 \\
\hline 10.0 & 7.0 & 1.0 & 50 & 1.64 \\
\hline 10.0 & 8.0 & 1.0 & 50 & 1.84 \\
\hline 10.0 & 5.0 & 0.8 & 50 & 3.03 \\
\hline 10.0 & 5.0 & 0.9 & 50 & 2.56 \\
\hline 10.0 & 5.0 & 1.0 & 50 & 1.97 \\
\hline 10.0 & 5.0 & 1.1 & 50 & 1.73 \\
\hline 10.0 & 5.0 & 1.2 & 50 & 1.51 \\
\hline 10.0 & 5.0 & 1.0 & 30 & 2.67 \\
\hline 10.0 & 5.0 & 1.0 & 40 & 2.53 \\
\hline 10.0 & 5.0 & 1.0 & 50 & 1.97 \\
\hline 10.0 & 5.0 & 1.0 & 60 & 1.78 \\
\hline 10.0 & 5.0 & 1.0 & 70 & 1.56 \\
\hline
\end{tabular}

Table 2. Effect of [Mn(II)], [acrylonitrile], $\left[\mathrm{Na}_{2} \mathrm{SO}_{4}\right]$ on the reaction rate at $308 \mathrm{~K}$

\begin{tabular}{|l|c|c|c|}
\hline $\begin{array}{l}{[\mathrm{Mn}(\mathrm{II})]} \\
\left(\mathrm{mol} \mathrm{dm}^{-3}\right)\end{array}$ & $\begin{array}{c}\text { Acrylonitrile] } \\
\left(\mathrm{mol} \mathrm{dm}^{-3}\right)\end{array}$ & $\begin{array}{c}{\left[\mathrm{Na}_{2} \mathrm{SO}_{4}\right]} \\
\left(\mathrm{mol} \mathrm{dm}^{-3}\right)\end{array}$ & $\begin{array}{c}10^{4} \mathrm{k}_{\text {obs }} \\
\left(\mathrm{s}^{-1}\right)\end{array}$ \\
\hline 0 & - & - & 1.97 \\
\hline 0.02 & - & - & 1.88 \\
\hline 0.04 & - & - & 1.81 \\
\hline 0.06 & - & - & 1.79 \\
\hline 0.08 & - & - & 1.75 \\
\hline- & 0 & - & 1.97 \\
\hline- & 0.02 & - & 2.38 \\
\hline- & 0.04 & - & 2.47 \\
\hline- & 0.06 & - & 2.28 \\
\hline- & 0.08 & - & 2.12 \\
\hline- & - & 0 & 1.97 \\
\hline- & - & 0.02 & 1.86 \\
\hline- & - & 0.04 & 2.07 \\
\hline- & - & 0.06 & 2.03 \\
\hline- & - & 0.08 & 1.89 \\
\hline
\end{tabular}

[Anil] $=0.08 \mathrm{~mol} \mathrm{dm}^{-3} ;$ [MMPP] $=0.005 \mathrm{~mol} \mathrm{dm}^{-3} ;\left[\mathrm{H}_{2} \mathrm{SO}_{4}\right]=$

$1.0 \mathrm{~mol} \mathrm{dm}{ }^{-3} ; \mathrm{HOAc}-\mathrm{H}_{2} \mathrm{O}=50 \%(\mathrm{v} / \mathrm{v})$; Temperature $=308 \mathrm{~K}$ 
Table 3. Temperature effect and thermodynamic parameters of MMPP oxidation of anils (substituents in aniline, benzaldehyde, both in aniline and benzaldehyde moiety)

\begin{tabular}{|c|c|c|c|c|c|c|c|c|c|c|c|c|c|}
\hline \multirow[b]{2}{*}{$\frac{\overrightarrow{0}}{\frac{0}{0}}$} & \multirow[b]{2}{*}{$\dot{z}$} & \multirow[b]{2}{*}{ Substituents } & \multicolumn{5}{|c|}{$10^{-4} \times \mathrm{k}_{\mathrm{obs}}\left(\mathrm{s}^{-1}\right)$} & \multirow[b]{2}{*}{$\begin{array}{c}\Delta \mathrm{H}^{\#} \\
\mathrm{~kJ} \mathrm{~mol}^{-1}\end{array}$} & \multirow{2}{*}{$\begin{array}{c}-\Delta \mathrm{S}^{\#} \\
\mathrm{~J} \mathrm{~K}^{-1} \mathrm{~mol}^{-1}\end{array}$} & \multirow[b]{2}{*}{$\begin{array}{c}\Delta \mathrm{G}^{\#} \\
\mathrm{~kJ} \mathrm{~mol}^{-1}\end{array}$} & \multirow[b]{2}{*}{$\begin{array}{c}\mathrm{E}_{\mathrm{a}} \\
\mathrm{kJ} \mathrm{mol}^{-1} \mathrm{~K}^{-1}\end{array}$} & \multirow[b]{2}{*}{$r$} & \multirow[b]{2}{*}{ SD } \\
\hline & & & $25^{\circ} \mathrm{C}$ & $30^{\circ} \mathrm{C}$ & $35^{\circ} \mathrm{C}$ & $40^{\circ} \mathrm{C}$ & $45^{\circ} \mathrm{C}$ & & & & & & \\
\hline \multirow{9}{*}{ 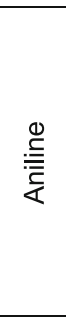 } & 1 & $\mathrm{H}$ & 1.59 & 1.72 & 1.97 & 2.30 & 3.07 & 226.48 & 242.29 & 301.10 & 228.86 & 0.961 & 0.07 \\
\hline & 2 & $\mathrm{~m}-\mathrm{CH}_{3}$ & 1.33 & 1.40 & 1.60 & 2.10 & 2.44 & 22.73 & 29.28 & 112.91 & 25.11 & 0.963 & 0.07 \\
\hline & 3 & $\mathrm{p}-\mathrm{CH}_{3}$ & 1.91 & 2.25 & 2.87 & 3.84 & 3.95 & 28.75 & 219.81 & 96.45 & 31.13 & 0.978 & 0.07 \\
\hline & 4 & $\mathrm{p}-\mathrm{OC}_{2} \mathrm{H}_{5}$ & 1.51 & 1.56 & 1.97 & 2.42 & 2.65 & 22.19 & 244.19 & 97.40 & 24.57 & 0.972 & 0.06 \\
\hline & 5 & $\mathrm{p}-\mathrm{OCH}_{3}$ & 1.38 & 1.49 & 1.73 & 1.42 & 1.72 & 3.51 & 306.93 & 98.04 & 5.89 & 0.374 & 0.10 \\
\hline & 6 & $\mathrm{p}-\mathrm{Cl}$ & 2.66 & 3.14 & 4.27 & 4.80 & 6.26 & 31.02 & 209.53 & 95.56 & 33.40 & 0.991 & 0.04 \\
\hline & 7 & $\mathrm{~m}-\mathrm{Cl}$ & 1.16 & 1.34 & 1.43 & 2.59 & 3.43 & 41.84 & 181.03 & 97.60 & 44.22 & 0.942 & 0.17 \\
\hline & 8 & $\mathrm{~m}-\mathrm{NO}_{2}$ & 1.87 & 2.22 & 2.55 & 2.35 & 2.64 & 9.22 & 285.00 & 97.00 & 11.60 & 0.806 & 0.07 \\
\hline & 9 & $\mathrm{p}-\mathrm{NO}_{2}$ & 1.52 & 2.07 & 2.16 & 2.35 & 4.07 & 30.25 & 216.71 & 97.00 & 32.63 & 0.909 & 0.16 \\
\hline \multirow{9}{*}{$\begin{array}{l}\frac{0}{0} \\
\stackrel{2}{0} \\
\frac{0}{0} \\
\frac{0}{\pi} \\
N \\
\frac{N}{0} \\
\infty\end{array}$} & 1 & $\mathrm{H}$ & 1.59 & 1.72 & 1.97 & 2.30 & 3.07 & 226.48 & 242.29 & 301.10 & 228.86 & 0.961 & 0.07 \\
\hline & 2 & $\mathrm{~m}-\mathrm{CH}_{3}$ & 1.43 & 1.57 & 1.84 & 2.21 & 3.17 & 27.71 & 226.31 & 97.41 & 30.09 & 0.957 & 0.09 \\
\hline & 3 & $\mathrm{p}-\mathrm{CH}_{3}$ & 1.73 & 2.06 & 2.90 & 3.90 & 4.90 & 40.17 & 182.62 & 96.42 & 42.55 & 0.994 & 0.04 \\
\hline & 4 & $\mathrm{p}-\mathrm{OC}_{2} \mathrm{H}_{5}$ & 1.29 & 1.41 & 1.64 & 2.08 & 3.41 & 33.83 & 206.93 & 97.56 & 36.21 & 0.932 & 0.15 \\
\hline & 5 & $\mathrm{p}-\mathrm{OCH}_{3}$ & 2.98 & 3.35 & 4.66 & 5.42 & 5.10 & 22.18 & 238.49 & 95.63 & 24.56 & 0.915 & 0.11 \\
\hline & 6 & $\mathrm{p}-\mathrm{Cl}$ & 1.71 & 2.24 & 2.62 & 3.14 & 2.64 & 16.65 & 260.46 & 96.87 & 19.03 & 0.809 & 0.13 \\
\hline & 7 & $\mathrm{~m}-\mathrm{Cl}$ & 1.23 & 1.37 & 1.80 & 2.06 & 3.59 & 37.39 & 195.28 & 97.54 & 39.77 & 0.947 & 0.14 \\
\hline & 8 & $\mathrm{~m}-\mathrm{NO}_{2}$ & 2.59 & 2.84 & 3.76 & 3.73 & 4.77 & 20.99 & 243.40 & 95.96 & 23.38 & 0.959 & 0.07 \\
\hline & 9 & $\mathrm{p}-\mathrm{NO}_{2}$ & 1.75 & 2.85 & 3.50 & 5.62 & fast & 103.39 & 27.58 & 94.90 & 105.77 & 0.898 & 0.58 \\
\hline \multirow{9}{*}{ 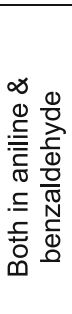 } & 1 & $\mathrm{H}$ & 1.59 & 1.72 & 1.97 & 2.30 & 3.07 & 226.48 & 242.29 & 301.10 & 228.86 & 0.961 & 0.07 \\
\hline & 2 & $\mathrm{~m}-\mathrm{CH}_{3}$ & 1.27 & 1.45 & 1.48 & 1.89 & 2.18 & 18.50 & 257.77 & 97.89 & 20.88 & 0.960 & 0.06 \\
\hline & 3 & $\mathrm{p}-\mathrm{CH}_{3}$ & 2.57 & 3.45 & 3.99 & 4.27 & 4.76 & 20.34 & 244.92 & 95.77 & 22.72 & 0.955 & 0.07 \\
\hline & 4 & $\mathrm{p}-\mathrm{OC}_{2} \mathrm{H}_{5}$ & 1.65 & 1.66 & 2.20 & 3.03 & 3.62 & 31.68 & 212.05 & 97.0 & 34.06 & 0.964 & 0.10 \\
\hline & 5 & $\mathrm{p}-\mathrm{OCH}_{3}$ & 2.57 & 3.18 & 3.53 & 4.28 & 5.11 & 23.71 & 234.16 & 95.83 & 26.09 & 0.994 & 0.02 \\
\hline & 6 & $\mathrm{p}-\mathrm{Cl}$ & 1.26 & 1.36 & 4.42 & 4.47 & 5.00 & 20.34 & 244.92 & 95.77 & 22.72 & 0.955 & 0.07 \\
\hline & 7 & $\mathrm{~m}-\mathrm{Cl}$ & 1.85 & 2.27 & 2.88 & 3.10 & 4.37 & 29.34 & 218.11 & 96.52 & 31.72 & 0.981 & 0.06 \\
\hline & 8 & $\mathrm{~m}-\mathrm{NO}_{2}$ & 1.99 & 2.51 & 2.96 & 3.95 & 4.51 & 30.31 & 214.17 & 96.27 & 32.69 & 0.994 & 0.03 \\
\hline & 9 & $\mathrm{p}-\mathrm{NO}_{2}$ & 0.86 & 1.30 & 1.34 & 1.58 & 3.44 & 44.02 & 175.41 & 98.05 & 46.40 & 0.912 & 0.22 \\
\hline
\end{tabular}

$\left[\right.$ Anil] $=0.1 \mathrm{~mol} \mathrm{dm}^{-3} ;$ MMPP] $=0.005 \mathrm{~mol} \mathrm{dm}^{-3} ;\left[\mathrm{H}_{2} \mathrm{SO}_{4}\right]=1.0 \mathrm{~mol} \mathrm{dm}^{-3} ; \mathrm{HOAc}-\mathrm{H}_{2} \mathrm{O}=50 \%(\mathrm{v} / \mathrm{v})$.

substituents in the aniline moiety, whereas the non-linear concave upward curve is observed for the substituents in benzaldehyde moiety and a non-linear concacve upward curve is observed for the substituents in the combination of aniline and benzaldehyde moiety.

\section{MECHANISM}

The addition of acrylonitrile monomer showed no variation in the reaction rate and no formation of turbidity (no formation of polymer) in the oxidation of aromatic anils. This rules out the presence of free radical mechanism in this oxidation reaction. So the ionic reaction is preferred in this oxidation study. Generally, the enhancement of the electrophillic activity of peroxide will minimize the importance of undesirable free radical pathways, resulting in a mixture of products ${ }^{\mathbf{1 6}}$. Usually peroxy ions act as strong nucleophiles. The absorption spectra represent spectral changes supporting the complex formation. The investigation results illustrate a fascinating note that the increase of $\mathrm{H}^{+}$retards the rate of the reaction. With the decrease of $\mathrm{pH}$, the molecule gains a proton, becomes less negatively charged and hence the rate of the reaction decreases ${ }^{17}$. Also in strong acid, the anil exists as $\mathrm{C}_{6} \mathrm{H}_{5} \mathrm{CH}={ }^{+} \mathrm{NHC}_{6} \mathrm{H}_{5}$ form. It is observed that when the acid strength is increased, there is a decrease in the rate which may be due to the less reactive protonated form than non-protonated form. Based on the stoichiometry of the reaction and above experimental results, the following reactions (Scheme 1) are believed to constitute the most probable mechanism of the reaction. The formation of complex is in agreement with the literature study ${ }^{\mathbf{9}, 18}$ and the intermediate formed is experimentally confirmed at $790 \mathrm{~nm}$ (Fig. 3). The next

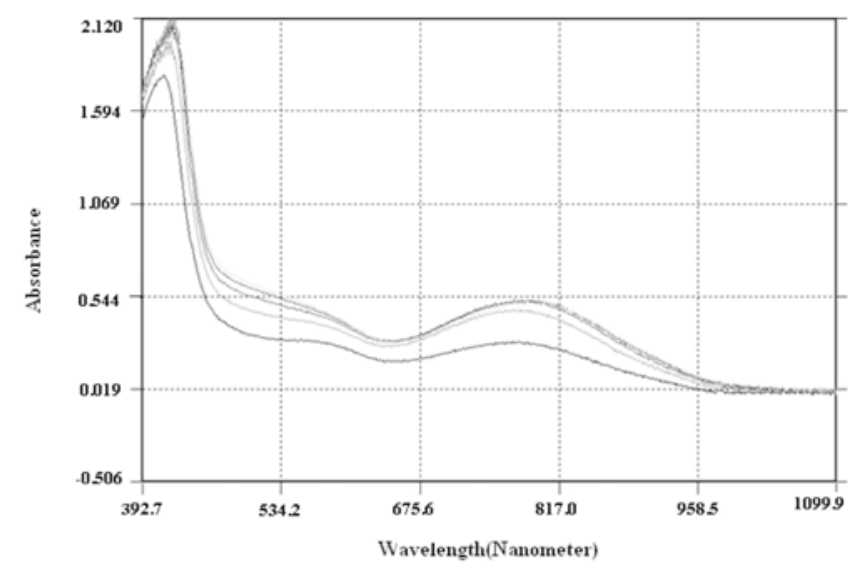

Figure 3. UV spectrum showing a probable formation of intermediate complex in oxidation of aromatic anil by MMPP

step (decomposition of complex) is a slow reaction and is likely to be the rate determining step. Simultaneously, at $429 \mathrm{~nm}$, a peak appears and is stable till the end of the reaction, which is the azobenzene product. Based on the above experimental observations, a probable mechanism, shown in Scheme 1 is suggested.

The above mechanism leads to the following rate law: Rate $=-\mathrm{d}[\mathrm{MMPP}] / \mathrm{dt}=\mathrm{K}_{1} \mathrm{k}_{2}$ [anil] [MMPP]

This rate law satisfactory explains all the experimental results.

\section{EFFECT OF SUBSTITUENTS ON THE REACTION RATE}

The present investigation, the kinetics of the oxidation of some meta- and para-substituted aromatic anils with MMPP was followed at five different temperatures 298, 


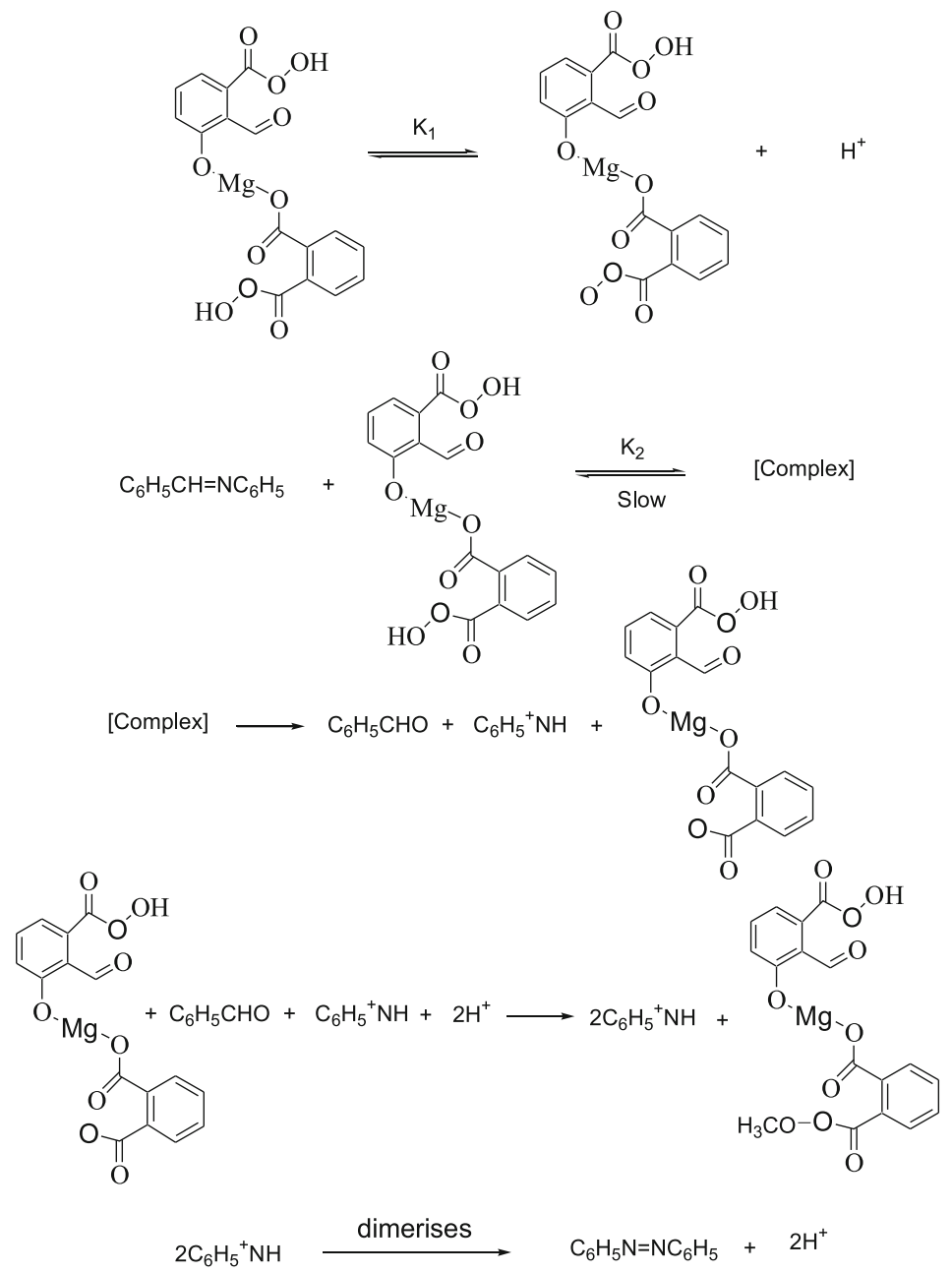

Scheme 1. Probable mechanism for the oxidation of aromatic anil by MMPP

$303,308,313$ and $318 \mathrm{~K}$ (Table 3), $\Delta \mathrm{H}^{\#}$ and $\Delta \mathrm{S}^{\#}$ values are calculated from the slope and intercept of the plot $\ln \mathrm{k}_{\mathrm{obs}} / \mathrm{T}$ versus $1 / \mathrm{T}$, respectively (Figs. 4, 5 and 6). The effect of substituents on the rate is studied by varying the substituents $\mathrm{H}, m-\mathrm{CH}_{3}, p-\mathrm{CH}_{3}, p-\mathrm{OC}_{2} \mathrm{H}_{5}, p-\mathrm{OCH}_{3}$, $p-\mathrm{Cl}, m-\mathrm{Cl}, m-\mathrm{NO}_{2}$ and $p-\mathrm{NO}_{2}$ in one of the rings benzaldehyde (or) aniline.

\section{DEVIATION FROM THE HAMMETT RELATIONSHIP}

\section{HAMMETT PLOT FOR ANILINE MOIETY}

Hammett made a unique discovery ${ }^{19}$ about the linear free energy relationships for the side chain. Application of the Hammett equation with the usual substituent constant $\sigma$ to the log kobs data of the meta- and para- substituted aromatic anils resulted in a concave downward curve (Fig. 7). Similar types of non-linear Hammett plots were observed previously in some reaction kinetics ${ }^{20}$. The non-linear concave downward curve is obtained for the anils with substituents in aniline moiety. The electron-releasing substituents fall on the one side of the curve with a positive slope and the electron-withdrawing substituents on the other side with a negative slope. The isokinetic plot and Exner plot reveal that there is no change in reaction mechanism with respect to substituents in aniline moiety. meta- and para- substituted anils with substituents in aniline moiety in aqueous acetic acid at $298 \mathrm{~K}-318 \mathrm{~K}$ confirm the Exner relationship, also the activation parameters to the isokinetic relationship but not to any of the linear free energy relationships. The

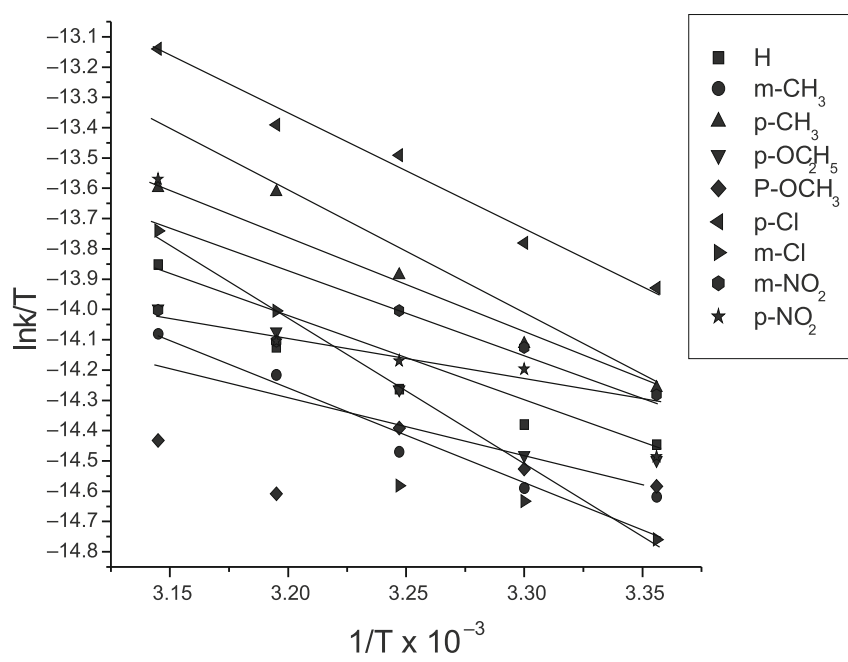

Figure 4. Eyring's plot for MMPP oxidation of anils (substitution in aniline moiety) at $308 \mathrm{~K}$

isokinetic temperature lies within the experimental range. The values of the negative and positive $\varrho$ values, $\mathrm{Q}+$ and $\mathrm{Q}^{-}$, respectively, at different temperature are given in Table 4. The unsubstituted anil is the most reactive in this series. Since the electron releasing substituents also retard the rate of the reaction, the rate-determining step proceeds with the development of negative charge on the nitrogen atom of anil. It is to be noted that this kind of transition state has been suggested in the oxidation of anil by INDC ${ }^{\mathbf{1 2}}$. Gurunathan Ramalingam and Sampath Kumar Jayanthi ${ }^{12}$ have established the order dependence with respect to the reactants and other 


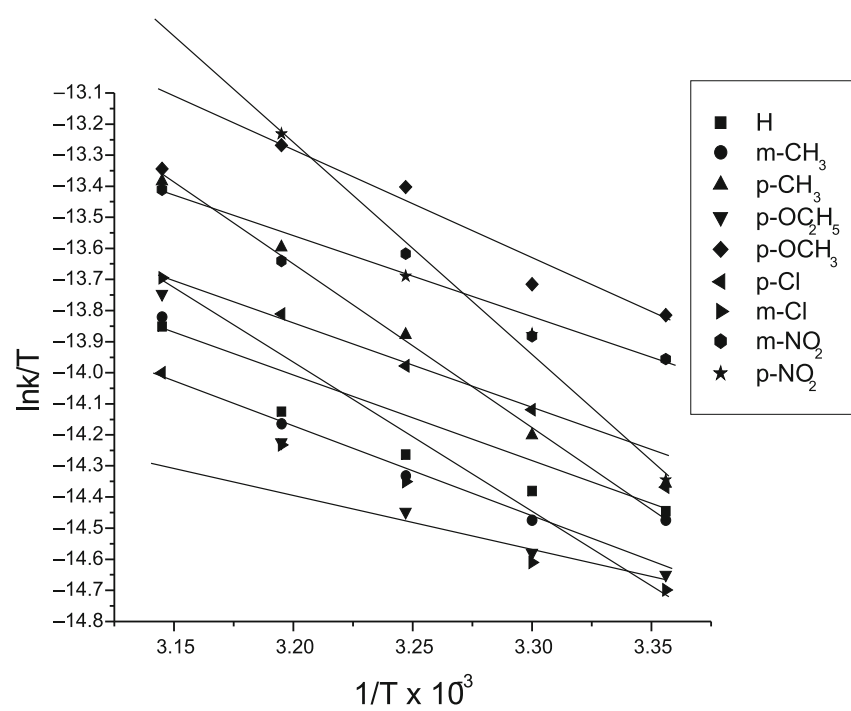

Figure 5. Eyring's plot for MMPP oxidation of anils (substitution in benzaldehyde moiety) at $308 \mathrm{~K}$

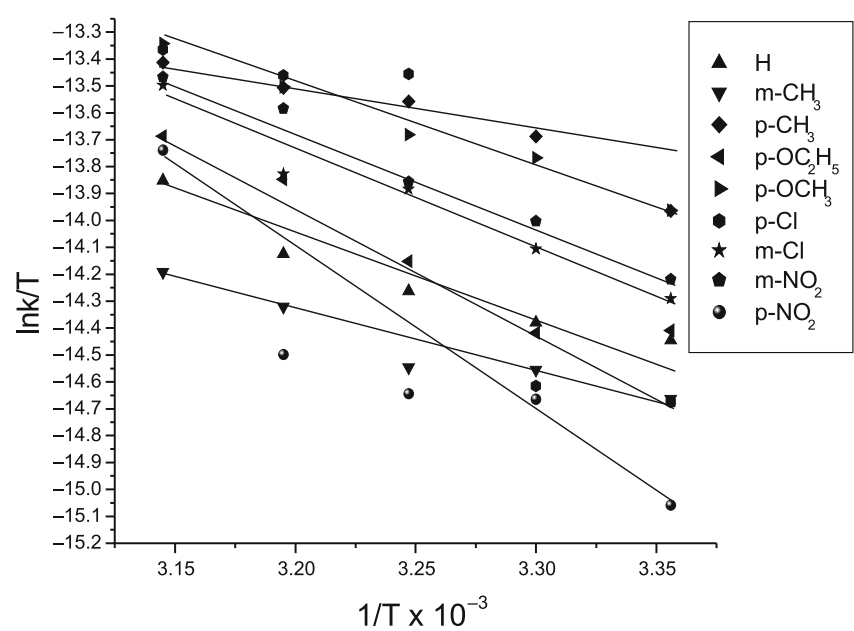

Figure 6. Eyring's plot for MMPP oxidation of anils (substitution in combination of aniline moiety and benzaldehyde moiety) at $308 \mathrm{~K}$

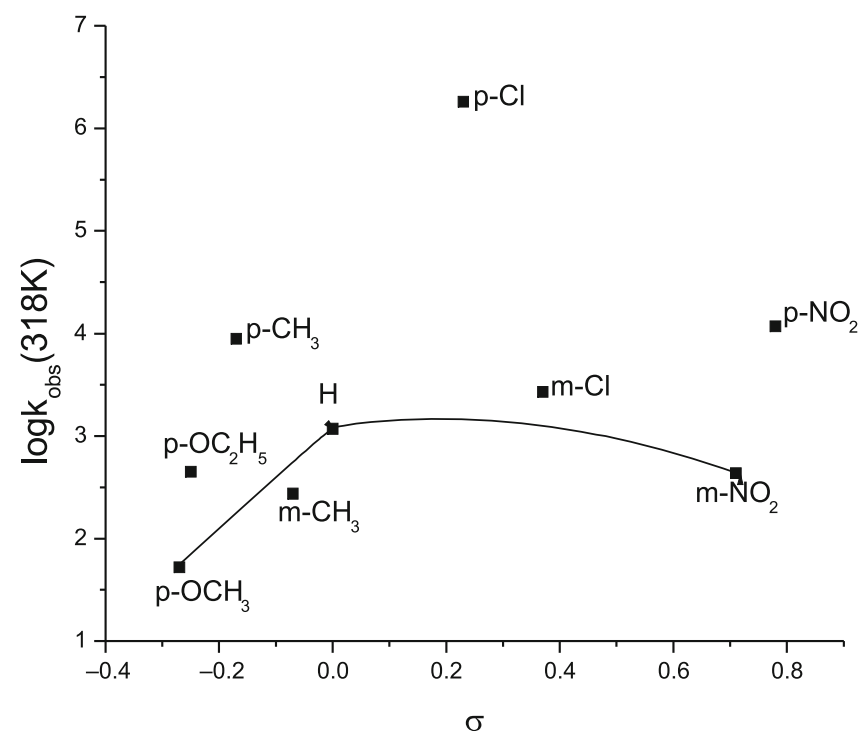

Figure 7. Hammett plot for MMPP oxidation of aromatic anils (substitution in aniline moiety) at $318 \mathrm{~K}$

kinetic parameters of anils (substitution only in aniline moiety). The formation of oxalatochromate species is reported as the intermediate of the reaction since oxalic acid is used as catalyst.
Table 4. Reaction constant for the potassium peroxymonosulfate oxidation of aromatic anils (substitutents in aniline, benzaldehyde, combination of aniline and benzaldehyde moiety)

\begin{tabular}{|l|c|c|c|}
\hline Substitutents & $\begin{array}{c}\text { Temperature } \\
(\mathrm{K})\end{array}$ & $\rho^{+}$ & $\rho^{-}$ \\
\hline \multirow{4}{*}{ Aniline moiety } & 298 & 0.103 & 0.289 \\
\cline { 2 - 4 } & 303 & 0.131 & 0.055 \\
\cline { 2 - 4 } & 308 & 0.339 & 0.392 \\
\cline { 2 - 4 } & 313 & 0.893 & 1.052 \\
\cline { 2 - 4 } Benzaldehyde moiety & 318 & 2.476 & 0.887 \\
\cline { 2 - 4 } & 298 & 1.701 & 1.437 \\
\cline { 2 - 4 } & 303 & 1.934 & 1.565 \\
\cline { 2 - 4 } & 308 & 2.058 & 1.791 \\
\cline { 2 - 4 } Combination of aniline \& & 313 & 2.339 & 1.933 \\
\cline { 2 - 4 } benzaldehyde moiety & 318 & 3.610 & 2.634 \\
\cline { 2 - 4 } & 298 & 1.461 & 0.228 \\
\cline { 2 - 4 } & 303 & 1.669 & 0.279 \\
\cline { 2 - 4 } & 308 & 1.832 & 1.034 \\
\cline { 2 - 4 } & 313 & 2.094 & 0.525 \\
\cline { 2 - 4 } & 318 & 2.620 & 0.301 \\
\hline
\end{tabular}

\section{HAMMETT PLOT FOR BENZALDEHYDE MOIETY}

The non-linear concave upward curve is obtained for the anils with substituents in benzaldehyde moiety (Figure 8). The electron-releasing substituents fall on the one side of the curve with a negative slope and the electron-withdrawing substituents on the other side with a positive slope. The isokinetic plot and Exner plot reveals that there is no change in the reaction mechanism with respect to the substituents in benzaldehyde moiety. The values of $\mathrm{Q}^{+}$and $\mathrm{Q}^{-}$at five different temperatures are given in Table 4. The unsubstituted anil is also the less reactive in this series. But both the electron-releasing and withdrawing substituents accelarate the rate of the reaction considerably dissimilar to aniline moiety. This shows the opposite kind of behaviour compared with aniline moiety.

\section{HAMMETT PLOT FOR COMBINATION OF ANILINE AND BENZALDEHYDE MOIETY}

The non-linear concave upward curve is obtained for the anils with substituents in combination of aniline and benzaldehyde moiety (Fig. 9) and the electron-releasing substituents fall on the one side of the curve with a negative slope and the electron-withdrawing substituents on the other side with a positive slope like in substituents

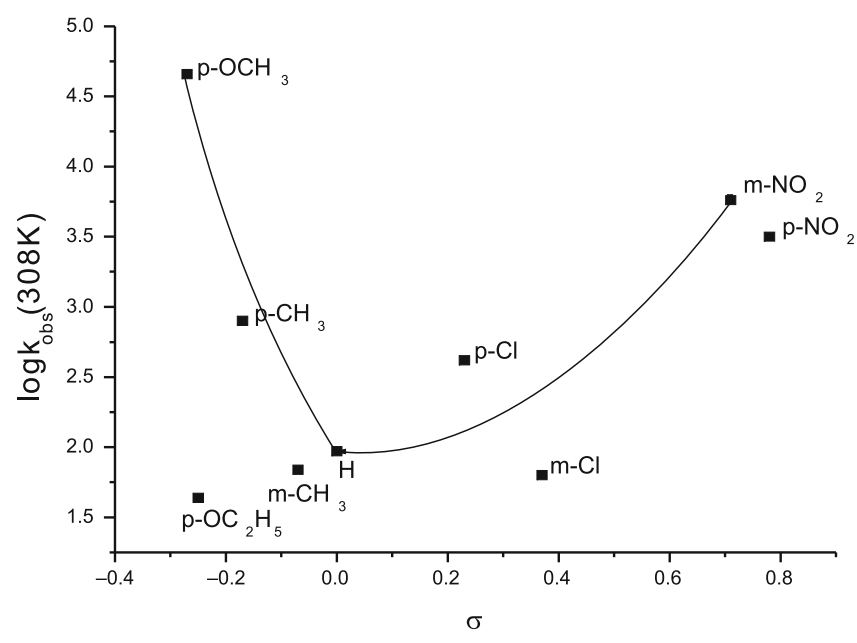

Figure 8. Hammett plot for MMPP oxidation of aromatic anils (substitution in benzaldehyde moiety) at $308 \mathrm{~K}$ 


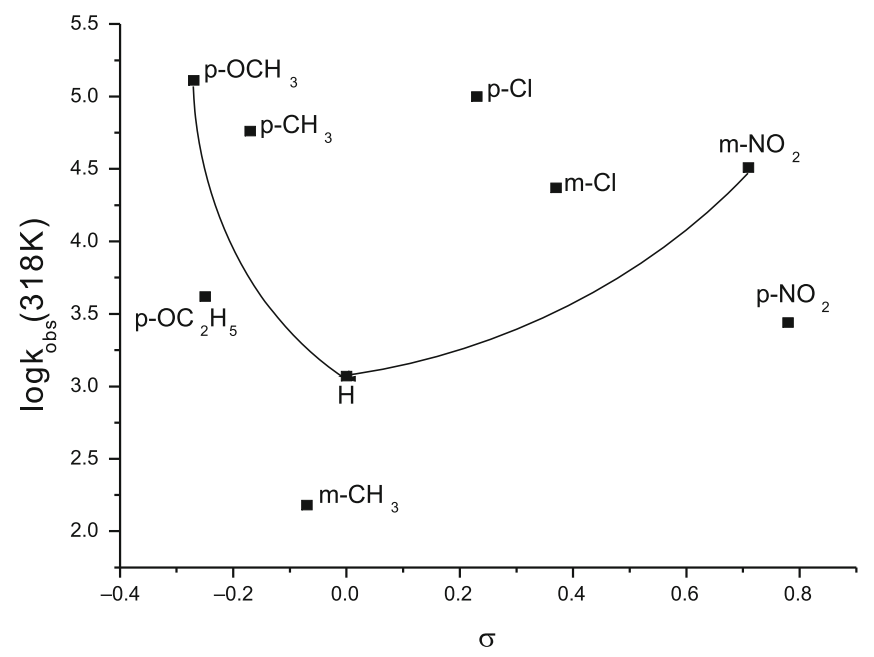

Figure 9. Hammett plot for MMPP oxidation of aromatic anils (substitution in combination of aniline and benzaldehyde moiety) at $318 \mathrm{~K}$

only in benzaldehyde moiety. The values of $\mathrm{Q}^{+}$and $\mathrm{Q}^{-}$at five different temperatures are given in Table 4.

\section{CONCLUSIONS}

The oxidation of aromatic anils by MMPP is the second order with respect to [anil] and is the first order with respect to MMPP and is the first order with respect to [acid] under the experimental conditions, and the aromatic anil is oxidized to benzaldehyde and azobenzene. The high negative entropy of activation, suggesting the formation of a complex is a slow step. Non-linear concave downward curve of Hammett plot is obtained for the anils with substituents in the aniline moiety whereas the non-linear concave upward curve is observed for the substituents in the benzaldehyde moiety and for the substituents in the combination of aniline and benzaldehyde moiety. The other methods available for the oxidation of anils are UV Spectrophotometry, Thermogravimetric analysis (TGA) and Differential thermal analysis (DTA). Compare to these methods, iodometric method is found to be simple, accurate and inexpensive. So, we have chosen iodometric method.

\section{LITERATURE CITED}

1. Brougham, P., Cooper, M.S., Cummerson, D.A., Heaney, H. \& Thompson, N. (1987). Epoxidation studies of decalin-1, 4-dienones and related alcohols. Synthe., 1015-1017. DOI: $10.1055 / \mathrm{s}-1987-28153$.

2. Heaney, H. (1993). Oxidation reactions using magnesium monoperphthalate and urea hydrogen peroxide. Aldrichimica Acta., 26, 35-45.

3. Joao, F.S., Carvalho, M., Manuel Cruz Silva, M. \& Luisa Sa e Melo. (2009). Highly efficient epoxidation of unsaturated steroids using magnesium bis(monoperoxyphthalate) hexahydrate. J. Tetrahed., 65, 2773-2781. DOI: 10.1016/j.tet.2009.01.100.

4. Ali, M.H. \& Stevens, W.C. (1997). Sulfides to sulfoxides on silica gel supported magnesium monoperoxyphthalate (MMPP) in methylene chloride. Synthe., 764-768.

5. Enrico Baciocchi, Osvaldo Lanzalunga. \& Andrea Lapi. (1995). Formation of quinines in the iron porphyrin catalyzed oxidation of benzene and alkylbenzenes by magnesium monoperoxyphthalate. Tetrahedron lett., 36, 3547-3548. DOI: 10.1016/0040-4039(95)00554-P.
6. Simon J. Hayes, David W. Knight, Andrew W.T. Smith. \& Mark J.O'Halloran. (2010). On the curious oxidations of 2-furylethanols. Tetrahedron lett., 51, 720-723. DOI: 10.1016/j.tetlet.2009.11.119.

7. Lisa Y. Wu, Joseph K. Choi, Krit Y. Hatton. \& Clifford E. Berkman. (2010). A simple method for the oxidation of a-amino acid esters to a-oximino esters. Tetrahedron lett., 51, 402-403. DOI: 10.1016/j.tetlet.2009.11.045.

8. Raja, M. \& Karunakaran, K. (2012). meso-Tetraphenylporphyriniron(III) chloride catalyzed oxidation of aniline and its substituents by magnesium monoperoxyphthalate in aqueous acetic acid medium. Pol. J. Chem. Tech., in press.

9. Karthikeyan, G., Elango, K.P., Karunakaran, K. \& Balasubramanian, A. (1998). Kinetics and mechanism of oxidation of aromatic anils by quinolinium chlorochromate. Oxid Commun, 21(1), 51-54.

10. Umesh, N., Ramesh, S. \& Dodwad, S.S. (1997). Kinetics and mechanism of acid bromate oxidation of 2-hydroxy-1-naphthalidene anil. Asian J. Chem., 9 (1), 58-62.

11. Karunakaran, K., Nagarajan, S., Kanagavel, D., Jegadish, T.N., Palanisamy, P.N. \& Elango, K.P. (1997). Interactive free energy relationship on the oxidation of aromatic anils by phenyliodoso acetate. Oxid Commun., 29 (4), 576.

12. Ramalingam, G. \& Jayanthi, S. (2007). Mechanistic investigation of oxidation of aromatic anils by isonicotinium dichromate in acetic medium. A Kinetic Study. Transit Met Chem Springer 2007, 32, 475-480. DOI: 10.1007/s11243-007-0190-x.

13. Lide, David R., ed. (2006). CRC Handbook of Chemistry and Physics (87th ed.). Boca Raton, FL: CRC Press. ISBN 0-8493-0487-3.

14. Venkatesh, R. \& Karunakaran, K. (2013). Kinetic investigation of oxidation of aromatic anils by potassium peroxymonosulfate in aqueous acidic medium. Int. J. Chem. Kinet., 2013 in press.

15. Karunakaran, C. \& Palanysamy, P.N. (2001). Autocatalysis in the sodium perborate oxidation of aniline in acetic acid-ethylene glycol. J. Mol. Catal. A-Chem., 172, 9-17. DOI: Org/10.1016/s 1381- 1169(01)00113-3.

16. Chandramohan, G., Kalyansundharam, S. \& Renganathan, R. (2002). Oxidation of indole -3-acetic acid by peroxomonosulphate: A kinetic and mechanic study. Int. J. Chem. Kinet., 34, 569. DOI: 10.1002/kin.10060.

17. Kutti rani, S., Nirmal kumar, S., Crystal Y Wilson., Gopi, A. \& Eswaramoorthy, D. (2009). Oxidation of vanillin by peroxomonosulphate-thermodynamic and kinetic investigation. $J$. Ind. Eng. Chem., 15, 898-901. DOI: 10.1016/j.jiec.2009.09.020.

18. Karthikeyan, G., Elango, K.P. \& Karunakaran K. (1997). Correlation analysis of reactivity in the oxidation of aromatic anils by pyridinium flurochromate. J. Indian Chem. Soc., 74(10), 798-799.

19. Hammett, Louis, P. (1937). Hammett equation. J. Am. Chem. Soc., 59, 96. DOI:10.1021/ja01280a022.

20. Hoffmann, J., Klicnar, J., Sterba, V. \& Vecera M. Coll. (1970). Deviations from hammett correlations-concave down Y. Czech. Chem. Commun., 35, 1387, http://www.docstoc.com/ docs/28281427/Hammett-correlations-and-their-applicationsin-elucidating 